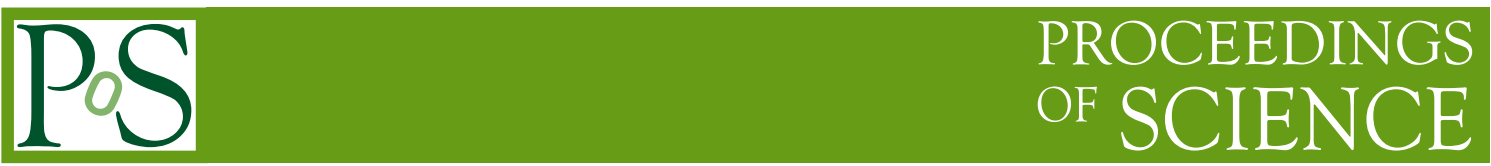

\title{
Secluded dark matter with a massive mediator
}

\author{
Shohei Okawa* \\ Nagoya University \\ E-mail: bkawadeken.phys.nagova-u.ac.ip
}

We study a dark matter (DM) model in which a DM particle interacts predominantly with nonSM particles (mediator particles) which decay into the SM particles later, while the DM particle has a very small coupling to the SM particles. This kind of DM is called secluded DM. We first introduce a simple model of secluded DM to survey the parameter space. Then, it is pointed out that if the mass splitting between DM and mediator is small, there is a novel thermal history of DM in which the DM number density evolution in early universe exhibits a temporary freezeout behavior. Besides, a larger annihilation cross section than that of well-known thermal relic DM models is required in order to achieve the observed density. Based on these observations, a novel possibility of DM model building is proposed in which DM and mediator are unified in an approximate dark symmetry multiplet. A pionic DM model is introduced to illustrate this idea in a renormalizable field theory framework. The model naturally realizes the degenerate mass spectrum and the large cross section of the DM and the mediator.

The 3rd International Symposium on "Quest for the Origin of Particles and the Universe" 5-7 January 2017

Nagoya University, Japan

\footnotetext{
* Speaker.
} 


\section{Introduction}

As one of promising DM scenarios consistent with the current experimental status, a secluded DM scenario has recently been proposed in Ref. [四]. In the secluded scenario, a DM particle annihilates predominantly into non-SM particles (mediator particles) subsequently decaying into the SM particles, unlike in usual scenarios the annihilation into the SM particles is dominant. In this work, we study a novel possibility in the secluded DM scenario that DM and mediator particles are almost degenerated in mass, which has not been discussed in the literature.

\section{A toy model}

\subsection{Setup}

To make our analysis concrete, let us introduce a simple model, with a DM particle $\phi_{d}$ and a mediator particle $\phi_{m}$,

$$
\begin{aligned}
\mathscr{L}= & \frac{1}{2}\left(\partial_{\mu} \phi_{d}\right)^{2}+\frac{1}{2}\left(\partial_{\mu} \phi_{m}\right)^{2}-\frac{1}{2} m_{d}^{2} \phi_{d}^{2}-\frac{1}{2} m_{m}^{2} \phi_{m}^{2} \\
& -\lambda_{1} \phi_{d}^{2}|\phi|^{2}-\lambda_{2} \phi_{m}^{2}|\phi|^{2}-\lambda_{3} \phi_{m}^{2} \phi_{d}^{2} \\
& -g m_{m} \phi_{m} \phi_{d}^{2},
\end{aligned}
$$

where the SM weak doublet Higgs field is denoted by $\phi$. The DM and mediator particles are real scalar and neutral under the SM gauge groups. The stability of the DM particle is guaranteed by a $Z_{2}$ symmetry, $\phi_{d} \rightarrow-\phi_{d}$, while the longevity of the mediator particle is not protected by the symmetry, due to the last term in Eq.(R. $(\mathbb{2})$. Note that we omit the terms consistent with the symmetry and renomalizability, such as $\phi_{d}^{2} \phi_{m}$, by hand. As one can see later, however, it is not crucial for our scenario whether we involve these terms in the model Lagrangian or not.

The original secluded proposal corresponds to a parameter choice of $m_{d} \gg m_{m}$ (and $\lambda_{1} \ll 1$ ). In this case, the mediator particle may behave as a part of thermal plasma until the DM density freezes out. Then, even if $\lambda_{1} \ll 1$ is assumed, the observed DM abundance can be achieved by appropriately choosing the annihilation cross section of the DM into the mediator. This possibility has already been studied well in the original secluded papers [ $[$ ]. Here we study a novel parameter region, $m_{d} \simeq m_{m}$ and $\lambda_{1} \ll 1$, which has not been investigated in the literature. Motivated by an unified description of the DM and mediator particles, we assume

$$
m_{d}=m_{m} \equiv m, \quad \lambda_{1}=\lambda_{2} \equiv \lambda(\ll 1),
$$

throughout this proceedings.

\subsection{An evolution example}

We show a example of the time evolution of the DM number density in Fig. I. The parameters are fixed at $m=1 \mathrm{TeV}, \lambda=1 \times 10^{-3}, \lambda_{3}=4$ and $g=2 \times 10^{-9}$ in the figure. The horizontal band is the observed value of the DM abundance within $1 \sigma$ by the Planck Collaboration, $\Omega_{C D M} h^{2}=$ $0.1188 \pm 0.0010$ [].

It can be seen that the DM density exhibits a "terrace" behavior in the thermal history unlike usual thermal relic DM scenarios, while the observed density can be achieved in fact. The unusual 
behavior is explained as follows. At high temperature $(z \ll 1)$, the DM and mediator particles keep in chemical equilibrium with the SM sector through the $\phi_{d(m)} \phi_{d(m)} \leftrightarrow \phi \phi^{\dagger}$ process, and their number densities trace the equilibrium ones. As temperature decreases down to $z \sim 20$, however, they chemically decouple from the SM sector; on the other hand, the chemical equilibrium between the DM and mediator particles still holds at this time because of the largeness of the coupling $\lambda_{3}$. Note that the mediator (inverse) decay process is not efficient for the thermalization with the SM sector, because the mediator lifetime is much longer than the Hubble time, i.e. $\tau_{m} \gg H(z \simeq 20)$.

For a while after the decoupling, the DM number density hardly changes, and behaves as if it freezes out. However, this behavior is temporary and the DM density starts to considerably decrease around $z \sim 800$. The trigger of the decrease is mediator decay. Once the mediator decay becomes active, the DM density also decreases along with that of the mediator. This is because the chemical equilibrium in the DM-mediator system forces the DM particles to rapidly convert to the mediator particles via the $\phi_{d} \phi_{d} \rightarrow \phi_{m} \phi_{m}$ process, so as to keep a balance between their number densities. Note that a large coupling $\lambda_{3}$ is required to keep the equilibrium at high $z$. As a result, the DM density decreases down to the observed one although the thermal history is quite unfamiliar.

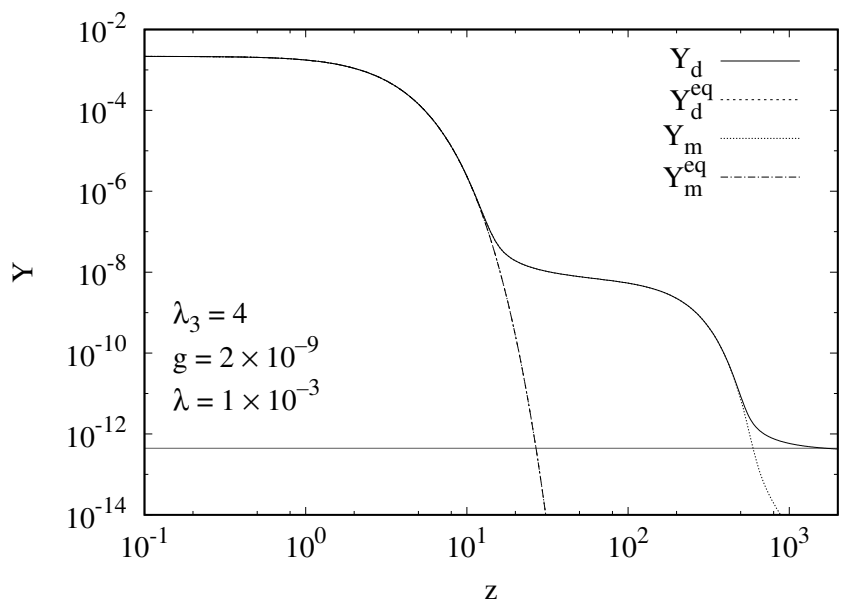

Figure 1: The evolution example of the DM number density with the fixed parameters; $m=1 \mathrm{TeV}, \lambda=$ $1 \times 10^{-3}, \lambda_{3}=4, g=2 \times 10^{-9}$. The horizontal axis is $z \equiv m_{d} / T$ and the vertical axis is the yields of the DM and mediator particles, $Y_{d(m)} \equiv n_{d(m)} / s$, where $s$ is the entropy density of the Universe.

\section{A renormalizable model}

Here we introduce a renomalizable model that implements the non-traditional thermal history of DM presented above. The DM and mediator particles are also unified in an approximate symmetry multiplet in this model.

The model Lagrangian consists of five parts,

$$
\mathscr{L}=\mathscr{L}_{S M}+\mathscr{L}_{D Q C D}+\mathscr{L}_{\text {yukawa }}+\mathscr{L}_{L \sigma M}+\mathscr{L}_{M},
$$

where $\mathscr{L}_{S M}$ is the SM Lagrangian involving the Higgs potential. We introduce a new confining gauge theory, dubbed dark quantum chromodynamics (DQCD),

$$
\mathscr{L}_{\mathrm{DQCD}}=-\frac{1}{4 g_{D s}^{2}} G_{\mu \nu}^{a} G^{a \mu \nu}+\bar{\psi} i D_{\mu} \gamma^{\mu} \psi
$$


with $\psi=(U, D)^{T}$ being dark quark fields in the fundamental representation, forming a dark chiral flavor doublet of $S U(2)$. We assume that the DQCD dynamics is analogous to the SM QCD. As will see below, candidates of DM and mediator particles show up as dark pions, pseudo-NambuGoldstone bosons resulting from the dynamical dark chiral symmetry breaking due to a dark quark pair condensate, $\langle\bar{\psi} \psi\rangle \neq 0$.

A linear sigma model is also introduced,

$$
\mathscr{L}_{L \sigma M}=\frac{1}{2}\left(\partial_{\mu} S\right)^{2}+\frac{1}{2}\left(\partial_{\mu} P\right)^{2}-V(S, P)
$$

with

$$
V=\frac{\lambda}{4}\left(S^{2}+P^{2}-F^{2}\right)^{2}+\frac{1}{4} \varepsilon_{\Delta} F^{2}\left(S^{2}-P^{2}\right)-\varepsilon_{S} F^{3} S-\varepsilon_{P} F^{3} P
$$

The first term in Eq.(B.4) preserves a $U(1)$ symmetry, while the second and third terms break it. The interactions between the SM and DQCD sectors are communicated by the Lagrangian,

$$
\mathscr{L}_{M}=-\left[y \bar{\psi}_{L}\left(S+i \tau^{3} P\right) \psi_{R}+\text { h.c. }\right]-\frac{\lambda_{M}}{2}\left(S^{2}+P^{2}\right)\left(\phi^{\dagger} \phi-v^{2} / 2\right),
$$

where $\phi$ and $v$ denotes the SM doublet Higgs field and the vacuum expectation value, respectively. Note that the first term in Eq.(B.5) also plays a role in a explicit dark chiral symmetry breaking, which makes the dark pions massive.

Assuming $v \ll \Lambda_{D Q C D} \ll F$ and integrating out the heavy degrees of freedom, we obtain the low energy effective Lagrangian described by the SM particles and the dark hadrons (pions); $\mathscr{L}_{\text {eff }}=$ $\mathscr{L}_{S M}+\mathscr{L}_{\chi, \text { eff. }}$ The relevant part in $\mathscr{L}_{\chi \text {,eff }}$ to our DM scenario is given by

$$
\begin{aligned}
\mathscr{L}_{\chi, \mathrm{eff}}= & \frac{1}{2} \partial_{\mu} \pi^{a} \partial^{\mu} \pi^{a}-\frac{1}{2} m_{\pi}^{2} \pi^{a} \pi^{a}+\lambda_{\pi \pi \phi^{\dagger} \phi} \pi^{a} \pi^{a} \phi^{\dagger} \phi+g_{\pi \phi^{\dagger} \phi} m_{\pi} \pi^{3} \phi^{\dagger} \phi \\
& -\frac{1}{6 f^{2}}\left[\left(\pi^{a} \partial_{\mu} \pi^{b}\right)^{2}-\left(\pi^{a} \partial_{\mu} \pi^{b}\right)\left(\pi^{b} \partial^{\mu} \pi^{a}\right)\right],
\end{aligned}
$$

with $f$ being the dark pion decay constant. It can be seen that $\pi^{1,2}$ and $\pi^{3}$ play the role of the DM and mediator particles, respectively, and both are actually degenerate in mass. The couplings, $\lambda_{\pi \pi \phi^{\dagger} \phi}$ and $g_{\pi \phi^{\dagger} \phi}$, which are expressed in terms of the parameters in the Lagrangian Eq.(B..J), correspond to $\lambda$ and $g$ in Sec.】, respectively. Note that the conversion process, $\phi_{d} \phi_{d} \rightarrow \phi_{m} \phi_{m}$, proceeds through the interaction term in the second line. Now we can find that all the significant terms are derived from the Lagrangian Eq.(B.]), assuming analogy of DQCD to SM QCD. For the detail of the analysis and further examples of the DM density evolution, see Ref. [B]].

\section{References}

[1] M. Pospelov, A. Ritz and M. B. Voloshin, Phys. Lett. B 662, 53 (2008); M. Pospelov and A. Ritz, Phys. Lett. B 671, 391 (2009); N. Arkani-Hamed, D. P. Finkbeiner, T. R. Slatyer and N. Weiner, Phys. Rev. D 79, 015014 (2009).

[2] P. A. R. Ade et al. [Planck Collaboration], arXiv:1502.01589 [astro-ph.CO].

[3] S. Okawa, M. Tanabashi and M. Yamanaka, Phys. Rev. D 95, no. 2, 023006 (2017) 